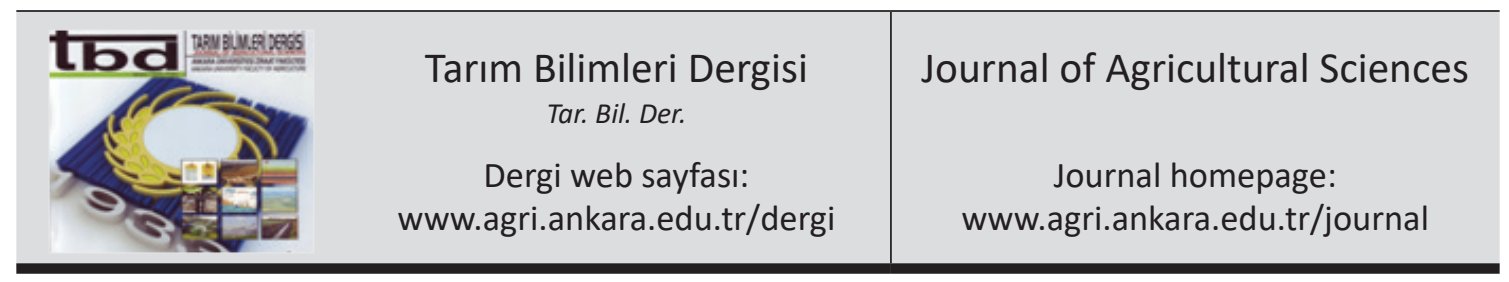

\title{
Rice Bran or Apple Pomace? Comparative Data Analysis of Astaxanthin Bioproduction
}

\author{
Derya DURSUN ${ }^{a}$, Ali Coşkun DALGIÇa \\ ${ }^{a}$ Gaziantep University, Department of Food Engineering, 27310, Gaziantep, TURKEY
}

\section{ARTICLE INFO}

Research Article

DOI: 10.15832 /ankutbd.456661

Corresponding Author: Derya DURSUN, E-mail: deryadursun@gantep.edu.tr, Tel: +90 (555) 8068131

Received: 11 April 2017, Received in Revised Form: 24 November 2017, Accepted: 28 November 2017

\begin{abstract}
Modeling and optimization of high value-added astaxanthin pigment bioproduction statistically by Sporidiobolus salmonicolor ATCC 24259 from two substantial wastes, rice bran (RB) and apple pomace (AP) was aimed in this study. The experimental data was obtained at constant inoculum rate $(2 \%)$ and particle size $(0.85 \mathrm{~mm})$ for both wastes by conducting 17 runs, which were generated by Box-Behnken design. $33.41 \mu \mathrm{g}$ astaxanthin $\mathrm{gRB}^{-}$and $77.31 \mu \mathrm{g}$ astaxanthin $\mathrm{gAP}^{-}$were produced as the maximum amount at the end of fermentation period, 10 days. Apple pomace was concluded as the optimized waste for the production of astaxanthin based upon the highest yield. Predicted response results of response surface methodology (RSM) and radial basis function-neural network (RBF-NN) were compared in order to evaluate the accuracy of two methodologies on non-linear behavior of the astaxanthin bioproduction. RBF-NN became prominent with its well-suited to apple pomace fermentation system by resulting in quite low 0.8495 , root mean square error (RMSE), 0.3349, mean absolute error (MAE), and 0.9985, correlation coefficient (CC) as best measures of a model performance.
\end{abstract}

Keywords: Apple pomace; Rice bran; Astaxanthin; Radial basis function-neural network

\section{Introduction}

The wastes of the cereals and fruits are obtained after post-harvesting and processing of them industrially, which are mayor columns of solid agro-industrial wastes. There are several methods for the waste management including composting, pyrolysis, combustion, gasification, land filling, animal feed etc. (López et al 2005; Arvanitoyannis 2010; Baino 2014; Panesar et al 2015). Bioprocesses are alternative and good way of utilization of the wastes, not just owing to their rich organic content, also for good anchorage feature, easy accessibility, abundantly available, low cost what kind of properties solid state fermentation (SSF) system requires (Pandey et al 2000; Couto \& Sanromán 2006; Couto 2008). SSF of the wastes is a highly attractive process to study due to providing environmental solutions of solid wastes, technique performance, wide usage and execution areas, feasibility, low cost and labor, product manifoldness and so on. However, the advantages in SSF are not efficient just for themselves. Also revealing certain fermentation conditions and productivity of the system is important by applying an experimental design, modeling and optimization statistically. In this manner, response surface methodology and artificial neural network (ANN) methodologies show 
up in order to evaluate the bio-processes. RSM is a methodology which uses the collection of numerical and statistical analyses by fitting the experimental data to a polynomial equation and describes the single and combination relations of the variables and responses mathematically and graphically as a result (Aslan \& Cebeci 2007; Baş \& Boyacı 2007). ANN is a mathematically modeling tool aimed to figure out complex non-linear relationships by simulating human brain learning process. Radial basis function is a particular data-based modeling neural network type that is frequently preferred to model bioprocesses. RBF-NN has three layers: an input layer, a hidden layer with non-linear RBF activation function, and a linear output layer. It introduces high-speed training rate, thus a quickly developed model is submitted (Warnes et al 1998; Dutta et al 2004). Consequently, the bio-processes could be improved, and productivity of the system may be increased by applying of these two strategies, RSM and RBF-NN as single or together to compare.

Rice bran and apple pomace as two of the most came out of the wastes have been used to produce bio-molecules such as ethyl alcohol, citric acid, microbial colors, enzymes etc. in SSF systems with different type of microorganisms (Laufenberg et al 2004; Joshi \& Attri 2006; Gupta et al 2011). Astaxanthin as a red color xanthophyll carotenoid is commercially produced as a targeted bio-molecule and used in preferentially food, feed, aquaculture, nutraceutical, pharmaceutical and poultry areas (Ambati et al 2014).

In this presented work, astaxanthin, which is very valuable carotenoid health wise, commercially and industrially, was chosen to be produce from rice bran and apple pomace by pure culture of Sporidiobolus salmonicolor. Box-Behnken experimental design was performed to obtain the experimental data, whereas RSM and RBF-NN methodologies were employed to get the predicted data. Comparison of the methodologies was carried out by several terms mathematically and statistically to reveal the predictive capability of them.

\section{Material and Methods}

\subsection{Wastes and microorganism culture}

RB and AP were supplied from Gaziantep and Adana provinces respectively. They were stored at cold storage $\left(+4{ }^{\circ} \mathrm{C}\right)$ in polyethylene packages. $\mathrm{RB}$ was directly sieved to size $0.85 \mathrm{~mm}$; AP was dried by tray drier, milled and then sieved in order to obtain a uniform material for fermentation system. Freeze-dried Sporidiobolus salmonicolor ATCC 24259 yeast was purchased from the American Type Culture Collection (Manassas, USA). The yeast was transformed in YM broth and maintained both in YM (Yeast-Malt) broth and agar. The composition of the media; $3 \mathrm{gL}^{-}$yeast extract (Merck, Germany), $3 \mathrm{gL}$ - malt extract (Merck, Germany), $5 \mathrm{~g} \mathrm{~L}$ - peptone (Merck, Germany), $10 \mathrm{~g} \mathrm{~L}^{-}$dextrose (SigmaAldrich, Germany) for the broth, and $20 \mathrm{~g} \mathrm{~L}^{-}$agar agar (Merck, Germany) for the agar were mixed and filled up with bi-distilled water. Fresh culture of ATCC 24259 was prepared by growing at its optimum growth conditions; $18.0^{\circ} \mathrm{C}$ and $6.0 \mathrm{pH}$ with regard to the ATCC protocol for 24 hours. Fresh culture at $2 \%\left(40.5 \mathrm{CFUg}^{-}\right)$inoculation rate was used for the inoculum.

\subsection{Extraction and spectral analysis}

A spectral scanning was done for astaxanthin (AX) standard (Chromadex, USA) which was purchased dissolved form in methanol and the wavelength of the maximum pick point was determined. Different concentrations (dilution series) of the AX standard with pure methanol (Sigma-Aldrich, Germany) were used to prepare standard curve. Spectrophotometric measurements of the series were carried out against pure methanol at the maximum wavelength. Standard curve of AX pigment was obtained using absorbance values versus the concentrations of the standard. Raw material and fermented content were subjected to pigment analysis. Sample was mixed with pure methanol at 1:4 ratio. The mixture was waited for 2 hours, $5 \mathrm{~mL}$ of the liquid phase of the mixture was taken and centrifuged at 6000 rpm for $10 \mathrm{~min}$. The supernatant was analyzed by double beam UV/VIS Spectrophotometer (Lambda 
25 UV/VIS Spectrophotometer, USA) against the pure methanol blank (Babitha et al 2007). AX concentration was calculated with regard to the equation of the standard curve. The results were explained as the mean of triplicate measurements and presented as microgram astaxanthin per gram dry waste $\left(\mu \mathrm{g} \mathrm{AX} \mathrm{gdw}^{-}\right)$.

\subsection{Modeling and optimization}

Response surface methodology in Design-Expert Version 7.1.5 (Minneapolis, USA) was used to generate an experimental design. Box-Behnken design (BBD) was selected using three independent variables; temperature $\left(\mathrm{x}_{1}\right)$, moisture content $\left(\mathrm{x}_{2}\right)$ and $\mathrm{pH}\left(\mathrm{x}_{3}\right)$ at three levels. Temperature and $\mathrm{pH}$ levels from ATCC protocol, and moisture content levels from water activity requirement $(0.60-$ 0.88 ) for the growth of the yeast were selected. The depended variable, response, was astaxanthin amount for the design. There were 17 runs with 5 center points conducted through the design (Table 1). Equation (1) introduces polynomial quadratic model as second order equation where $y$ is response or dependent variable; $\beta_{\mathrm{o}}$ is model constant; $\beta_{1}, \beta_{2}, \beta_{3}$ are linear coefficients; $\beta_{12}, \beta_{13}, \beta_{23}$ are cross product coefficients (present the interactions between the variables); $\beta_{11}, \beta_{22}, \beta_{33}$ are quadratic coefficients; independent variables: $x_{1}$ is temperature, $x_{2}$ is moisture content and $x_{3}$ is $\mathrm{pH}$. The predicted data was obtained from the analysis of RSM methodology.

$y=\beta_{0}+\beta_{I} x_{I}+\beta_{2} x_{2}+\beta_{3} x_{3}+\beta_{12} x_{T} x_{2}+\beta_{13} x_{T} x_{3}+\beta_{23} x_{2} x_{3}+\beta_{I I} x_{I I}+\beta_{22} x_{22}+\beta_{33} x_{33}$

The experimental data of BBD was exposed to Gausiian function with 0.75 spreadability in RBF$\mathrm{NN}$ in MATLAB Version 7.10 (USA) using the equation below (2) in order to estimate the data with 3 input layer-one hidden layer with 17 nodes-1 output layer (3-17-1) topology.

$a_{h k}=\exp \left(-\left\|x_{h}-x_{k}\right\|^{2} / \sigma_{h}^{2}\right)$

Where; $a_{h k}$ is basis function or activation of h-th unit in the hidden layer; $x_{h}$ is unit center or n-dimensional position of the center of $\mathrm{h}$-th ( $\mathrm{n}$ as input number); $x_{k}$, mean or center of RBF element, $\sigma_{h}$ is standard deviation or local scaling constant. Input variables as

Table 1- The design matrix and response results in RSM and RBF-NN

\begin{tabular}{ccccrrrrrr}
\hline \multirow{2}{*}{ Run } & $x_{1}$ & $x_{2}$ & $x_{3}$ & \multicolumn{9}{c}{$R B$} & $E$ & $R S M-P$ & $R B F-N N-P$ & $E$ & $R S M-P$ & $R B F-N N-P$ \\
\hline 1 & 18 & 70 & 7 & 4.28 & 8.15 & 4.28 & 6.72 & 1.13 & 6.72 \\
2 & 18 & 80 & 6 & 10.83 & 15.12 & 15.12 & 25.82 & 26.08 & 26.08 \\
3 & 23 & 70 & 6 & 7.95 & 4.22 & 7.95 & 10.99 & 19.61 & 10.99 \\
4 & 18 & 90 & 7 & 27.29 & 24.52 & 27.29 & 18.16 & 23.91 & 18.16 \\
5 & 13 & 80 & 5 & 9.73 & 9.87 & 9.73 & 31.78 & 34.81 & 31.78 \\
6 & 23 & 80 & 5 & 11.75 & 12.70 & 11.75 & 8.48 & 5.61 & 8.48 \\
7 & 18 & 80 & 6 & 9.86 & 15.12 & 15.12 & 26.94 & 26.08 & 26.08 \\
8 & 23 & 80 & 7 & 9.86 & 9.72 & 9.86 & 5.22 & 2.20 & 5.22 \\
9 & 13 & 80 & 7 & 8.92 & 7.97 & 8.92 & 24.74 & 27.61 & 24.74 \\
10 & 13 & 90 & 6 & 17.15 & 20.88 & 17.15 & 77.31 & 68.69 & 77.31 \\
11 & 18 & 70 & 5 & 5.23 & 8.01 & 5.23 & 13.17 & 7.43 & 13.17 \\
12 & 18 & 80 & 6 & 25.71 & 15.12 & 15.12 & 23.17 & 26.08 & 26.08 \\
13 & 18 & 80 & 6 & 13.41 & 15.12 & 15.12 & 26.82 & 26.08 & 26.08 \\
14 & 23 & 90 & 6 & 25.10 & 28.01 & 25.10 & 16.47 & 13.75 & 16.47 \\
15 & 13 & 70 & 6 & 9.69 & 6.77 & 9.69 & 16.55 & 19.27 & 16.55 \\
16 & 18 & 90 & 5 & 33.41 & 29.54 & 33.41 & 22.62 & 28.21 & 22.62 \\
17 & 18 & 80 & 6 & 15.79 & 15.12 & 15.12 & 27.65 & 26.08 & 26.08 \\
\hline
\end{tabular}

$E$, experimental data; $P$, predicted data 
the fermentation parameters $\left(\mathrm{x}_{1}, \mathrm{x}_{2}\right.$, and $\left.\mathrm{x}_{3}\right)$ and output variable as the astaxanthin amount (y) were utilized for the non-linear mapping in RBF-NN. Integration analyses of the experimental and the predicted data were performed to judge the quality of predictions by RMSE, MAE, CC, $\mathrm{R}^{2}$ and adjusted $\mathrm{R}^{2}$ measures, whose expressions were referred to Willmott (1982).

\subsection{Solid state fermentation}

100 gram total amount of the waste and bi-distilled water in $250 \mathrm{~mL}$ Erlenmeyer flask was prepared as fermentation content. Amount of the water to be added was determined depending on initial and last moisture content of the solid waste. $\mathrm{pH}$ value of the water to be according to the experimental design was adjusted by $\mathrm{HCl}$ (Merck, Germany) and $\mathrm{NaOH}$ (Merck, Germany) solutions. Water and solid waste was mixed in flasks, which were autoclaved (HMC HV-85L Autoclave, Germany) at $121.0^{\circ} \mathrm{C}$ for 15 minutes. After inoculation, incubation was maintained at the design temperature degrees during the fermentation period.

\section{Results and Discussion}

Bio-production of astaxanthin from RB and AP was accomplished by Sporidiobolus salmonicolor applying SSF technology. Experimental data obtained according to BBD matrix and predicted data generated by RSM and RBF-NN was presented in Table 1. Individual and interaction effects of the independent variables on the response were investigated well thanks to response surface plots, model equation coefficients and probability analysis in RSM by DoE. Therefore, the relationships between the solid state fermentation parameters and astaxanthin amount produced could be explained. The quadratic equations in the coded values for both wastes were given below.

RB quadratic equation with coded coefficients: $\mathrm{y}=15.1201+1.1456 x_{1}+9.4745 x_{2}-1.2215 x_{3}+2.4226 x_{1} x_{2}-$ $0.2715 x_{T} x_{3}-1.2909 x_{2} x_{3}-3.8191 x_{11}+3.6694 x_{22}-1.2364 x_{33}$

AP quadratic equation with coded coefficients: $\mathrm{y}=26.08-13.65 x_{1}-2.65 x_{2}+10.89 x_{3}+0.95 x_{1} x_{2}-$ $13.82 x_{1} x_{3}+0.5 x_{2} x_{3}+3.32 x_{11}-11.84 x_{22}+0.93 x_{33}$
Whereas the model was significant only at $\mathrm{P}<0.1$ for $\mathrm{RB}$ system, it was significant at all probability intervals for AP system as seen in Table 2. Lack of fit expression is expected as 'not significant', which is good for fitting of the model. It was observed that a reverse result for both fermentation systems at all probability intervals. Significant lack of fit result for AP system meant that there was a bad fitting. It could be also seen the optimized fermentation conditions through the experimental and predicted at the same table. The highest astaxanthin amount, $77.31 \mu \mathrm{g} \mathrm{AX} \mathrm{gdw}^{-}$was produced from the apple pomace system at the highest moisture content, lowest temperature and middle $\mathrm{pH}$ levels. It was understood that there was almost a perfect fitting between the experimental and predicted optimized results. However, lack of fit result for AP system had been commented as a bad fitting that caused the first confusion. In the mean time, $\mathrm{RB}$ system produced maximum $33.41 \mu \mathrm{g}$ AX $\mathrm{gdw}^{-}$at the highest moisture content level.

Table 2- Optimum RSM results for rice bran and apple pomace fermentation systems

\begin{tabular}{|c|c|c|c|c|c|}
\hline \multirow{2}{*}{\multicolumn{2}{|c|}{ Optimized conditions }} & \multicolumn{2}{|c|}{$R B$} & \multicolumn{2}{|c|}{$A P$} \\
\hline & & $E$ & $P$ & $E$ & $P$ \\
\hline \multicolumn{2}{|c|}{ Temperature $\left({ }^{\circ} \mathrm{C}\right)$} & 18.0 & 20.52 & 13.0 & 13.0 \\
\hline \multicolumn{2}{|c|}{ Moisture content $(\%)$} & 90.0 & 90.0 & 90.0 & 90.0 \\
\hline \multicolumn{2}{|l|}{$\mathrm{pH}$} & 5.0 & 5.0 & 6.0 & 5.87 \\
\hline \multicolumn{2}{|c|}{ Max yield ( $\mu \mathrm{g}$ AX gdw) } & 33.41 & 29.54 & 77.31 & 68.69 \\
\hline \multirow[t]{2}{*}{ Tools } & $\mathrm{P}<$ & \multicolumn{4}{|c|}{ Prob $>$ F } \\
\hline & 0.1 & & + & & + \\
\hline \multirow[t]{3}{*}{ Model } & 0.05 & -0. & 0995 & & 0.043 \\
\hline & 0.01 & & - & & + \\
\hline & 0.1 & & - & & + \\
\hline \multirow[t]{2}{*}{ Lack of fit } & 0.05 & -0. & 5726 & & 0.024 \\
\hline & 0.01 & & - & & + \\
\hline
\end{tabular}

Response surface plots (Figure 1 and 2) demonstrated the interaction of the fermentation parameters. It could be surely said that $\mathrm{pH}$ parameter had no effect on the bioproduction utilizing the wastes 

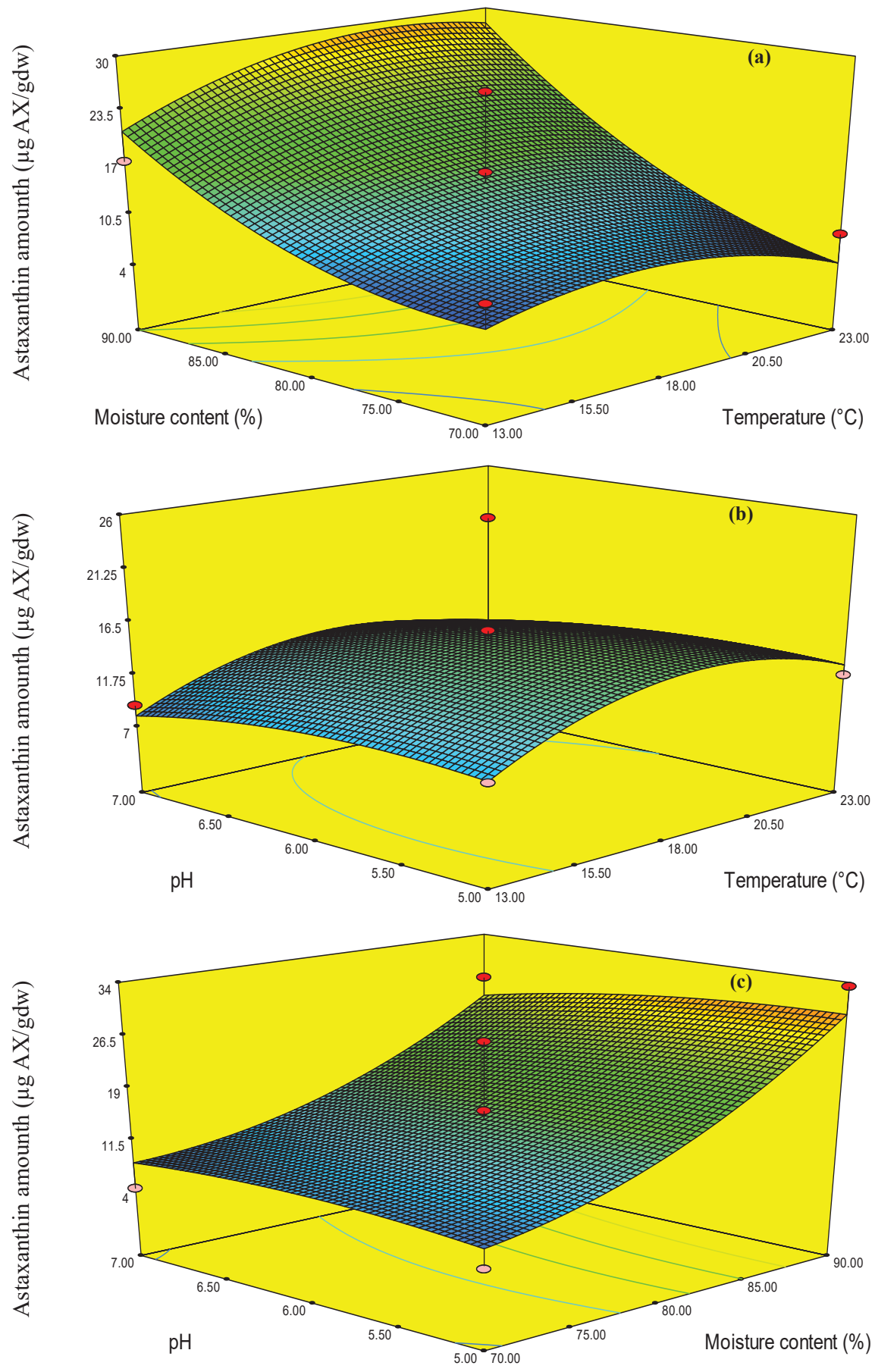

Figure 1- Response surface plots (a, b, c) of the interactions for RB fermentation system 

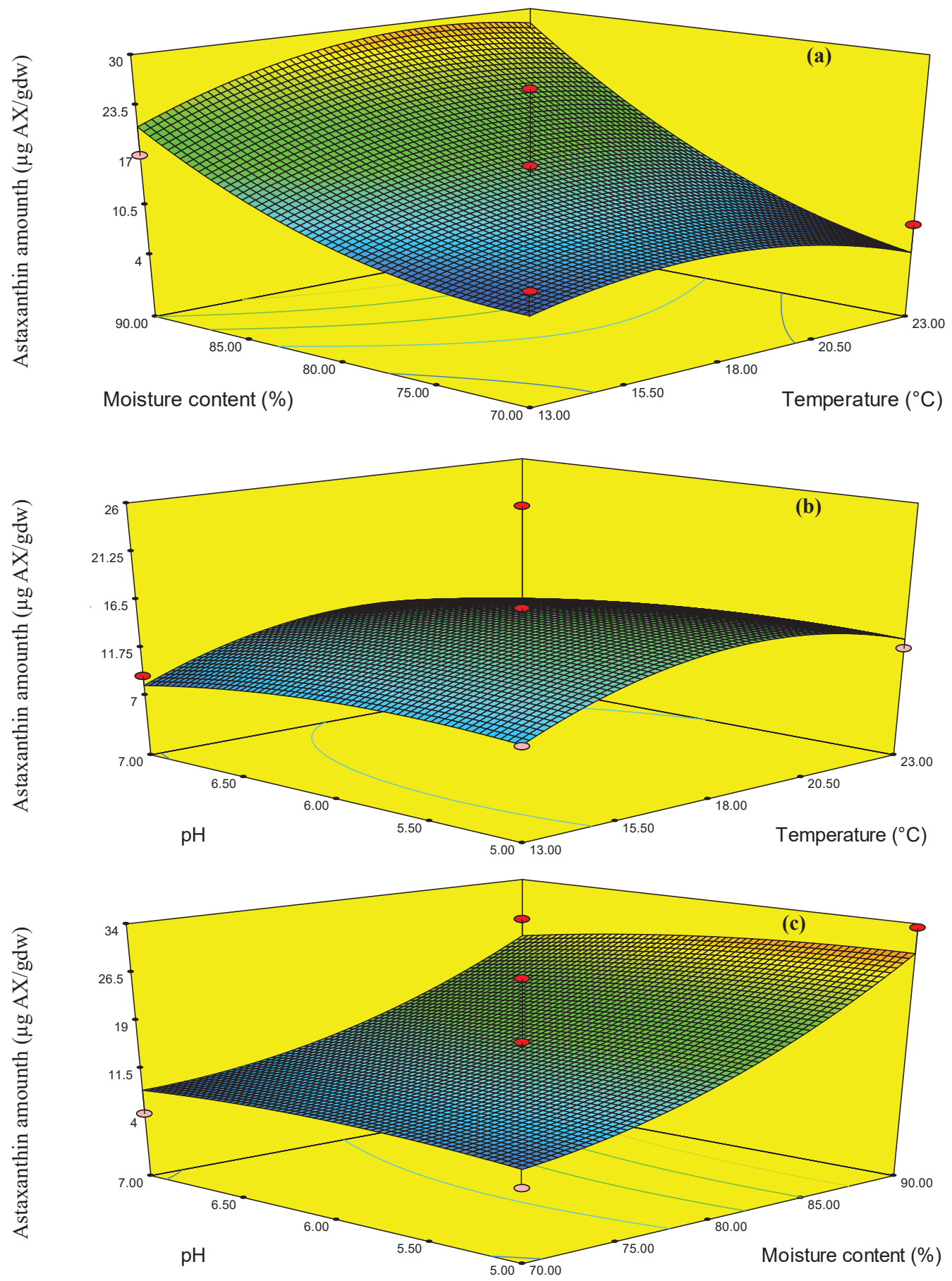

Figure 2- Response surface plots $(a, b, c)$ of the interactions for AP fermentation system 
as single or in company. Moisture content had the most significant effect on the astaxanthin production from both wastes. It was clearly seen that moisture content was a great inducer. Temperature parameter with moisture content showed that mild values of it had low significant effect. On the other hand, a slight increasing at temperature with $\mathrm{pH}$ meant significancy on both bioproductions. Prediction quality (or capacity) of two methodologies was needed to be revealed in terms of comparing, and also overcoming some incoherency. For these purposes, the parameters in Table 3 were estimated for each waste fermentation system. Small RMSE and MAE, high CC, $\mathrm{R}^{2}$ and Adj. $\mathrm{R}^{2}$ values indicates well approximation for predicting and exhibits good fitting. When the comparison results were evaluated between themselves and among each other, RBFNN showed more successful predictions for the solid state fermentation of the rice bran and apple pomace. Although $\mathrm{R}^{2}$ value, which means well replication of the experimental data to predict by the model, was much higher for AP system, the model fitting (lack of fit) had showed not-significant result at even all probability intervals. This caused the second confusion. It is known that RSM is a widely used and does a good job for microbiological, fermentation, biotechnological processes. However, the power and quality of RSM needed to be confirmed that this study brought out clearly. RBF$\mathrm{NN}$ as a local approximator offered us a superior prediction owing to high accuracy, particularly for AP system that was mathematically supported (Table 3). It has been indicated by many studies in literature that neural networks applications has a real superiority over RSM (Warnes et al 1998; Dutta

Table 3- Comparison of RSM and RBF-NN methodologies

\begin{tabular}{lcccc}
\hline \multirow{2}{*}{ Parameters } & \multicolumn{2}{c}{$R B$} & \multicolumn{2}{c}{$A P$} \\
\cline { 2 - 5 } & $R S M-P$ & $R B F-N N-P$ & $R S M-P$ & $R B F-N N-P$ \\
\hline RMSE & 3.8608 & 3.0819 & 4.4648 & 0.8495 \\
MAE & 2.6976 & 1.1857 & 3.3409 & 0.3349 \\
CC & 0.8943 & 0.9381 & 0.9591 & 0.9985 \\
$\mathrm{R}^{2}$ & 0.7783 & 0.8588 & 0.9198 & 0.9971 \\
Adj. $\mathrm{R}^{2}$ & 0.7636 & 0.8493 & 0.9145 & 0.9969 \\
\hline
\end{tabular}

et al 2004; Fang \& Horstemeyer 2007; Desai et al 2008; Liu et al 2009; Deshmukh et al 2012). Figures 3 and 4 depicted the comparison of generalization ability of RSM and RBF-NN for both wastes by regression measurements. They also substantiated the good predictor characteristic of RBF-NN.

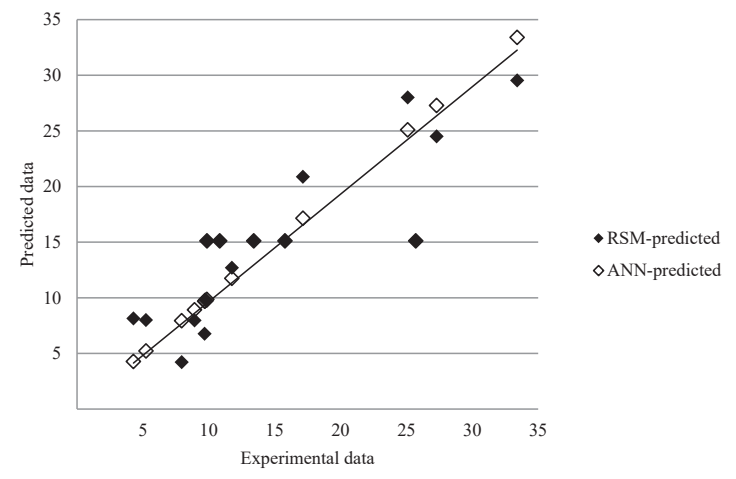

Figure 3- Comparative parity plots of RSM and RBF-NN data sets for $R B$

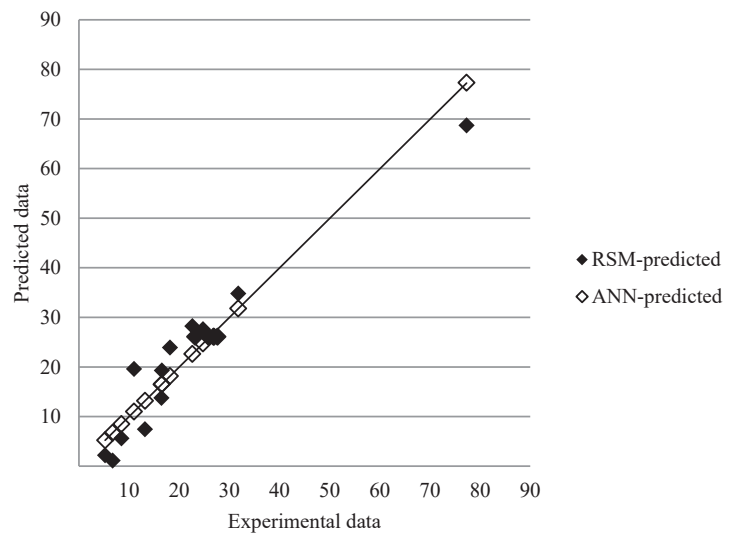

Figure 4- Comparative parity plots of RSM and RBF-NN data sets for AP

\section{Conclusions}

The following statements have been concluded from solid state fermentation of RB and AP by S. salmonicolor yeast: 1 . Optimizing the fermentation conditions and resource in the scope of an experimental design were achieved for a valuable bio-product, astaxanthin. 2. Moisture content was 
assigned as the most significant parameter for both waste fermentation systems. 3. Apple pomace was determined as a good resource due to resulting considerably high astaxanthin yield. 4. RSM and RBF-NN are both popular applications for the evaluation of fermentation systems statistically and mathematically, for which were performed in this study. Eventually, RBF-NN displayed a superior role on prediction of the original data set that several comparison parameters assisted. RBF-NN may be used substituted for RSM.

\section{Acknowledgements}

This study was funded by Scientific Research Foundation of Gaziantep University (BAP M.F.12.08 and BAP M.F.14.12).

\section{References}

Ambati R R, Phang S M, Ravi S \& Aswathanarayana R G (2014). Astaxanthin: sources, extraction, stability, biological and its commercial applications-A review. Marine Drugs 12(1): 128-152

Arvanitoyannis I S (2010). Waste Management for the Food Industries. Charon Tec Ltd, First Edition, Chennai, India

Aslan N \& Cebeci Y (2007). Application of Box-Behnken design and response surface methodology for modeling of some Turkish coals. Fuel 86(1): 90-97

Babitha S, Soccol C R \& Pandey A (2007). Solidstate fermentation for the production of Monascus pigments from jackfruit seed. Bioresource Technology 98(8): 1554-1560

Baino A (2014). Recovery of biomolecules from food wastes--a review. Molecules 19(9): 14821-14842

Baş D \& Boyacı I H (2007). Modeling and optimization I: Usability of response surface methodology. Journal of Food Engineering 78(3): 836-845

Couto S R (2008). Exploitation of biological wastes for the production of value-added products under solidstate fermentation conditions. Biotechnology Journal 3(7): $859-870$

Couto S R \& Sanromán M A (2006). Application of solid state fermentation to food industry- A review. Journal of Food Engineering 76: 291-302

Desai K M, Survase S A, Saudagar P S, Lele S S \& Singhal R S (2008). Comparison of artificial neural network (ANN) and response surface methodology (RSM) in fermentation media optimization: Case study of fermentative production of scleroglucan. Biochemical Engineering Journal 41(3): 266-273

Deshmukh S C, Senthilnath J, Dixit R M, Malik S N, Pandey R A, Vaidya A N, Omkar S N \& Mudliar S N (2012). Comparison of radial basis function neural network and response surface methodology for predicting performance of biofilter treating toluene. Journal of Software Engineering and Applications 5(8): 595-603

Dutta J R, Dutta P K \& Banerjee R (2004). Optimization of culture parameters for extracellular protease production from a newly isolated Pseudomona ssp. using response surface and artificial neural network models. Process Biochemistry 39(12): 2193-2198

Fang H \& Horstemeyer M F (2007). Global response approximation with radial basis functions. Engineering Optimization 38(4): 407-424

Gupta C, Garg A P, Prakash D, Goyal S \& Gupta S (2011). Microbes as potential source of biocolors. Pharmacology 2: 1309-1318

Joshi V K \& Attri D (2006). Solid state fermentation of apple pomace for the production of value added products. Natural Product Radiance 5(4): 289-296

Laufenberg G, Kunz B \& Rosato P (2004). Adding value to vegetable waste: oil press cakes as substrates for microbial decalactone production. European Journal of Lipid Science and Technology 106(4): 207-217

Liu L, Jun Sun J, Zhang D, Du G, Chen J \& Xu W (2009). Culture conditions optimization of hyaluronic acid production by Streptococcus zooepidemicus based on radial basis function neural network and quantumbehaved particle swarm optimization algorithm. Enzyme and Microbial Technology 44: 24-32

López S, Davies D R, Giráldez F J, Dhanoa M S, Dijkstra J \& France J (2005). Assessment of nutritive value of cereal and legume straws based on chemical composition and in vitro digestibility. Journal of the Science of Food and Agricultural 85(9): 1550-1557

Pandey A, Soccol C R \& Mitchell D (2000). New developments in solid state fermentation: I-bioprocesses and products. Process Biochemistry 35(10): 1153-1169

Panesar R, Kaur S \& Panesar P S (2015). Production of microbial pigments utilizing agro-industrial waste: a review. Current Opinion in Food Science 1: 70-76

Warnes M R, Glassey J, Montague G A \& Kara B (1998). Application of radial basis function and feed forward artificial neural networks to the Escherichia coli fermentation process. Neurocomputing 20: 67-82

Willmott C J (1982). Some comments on the evaluation of model performance. Bulletin of the American Meteorological Society 63(11): 1309-1313 